\title{
Steroidogenic Activity of Aldosterone-Producing Adenoma with and without KCNJ5 Gene Mutations, Comparing with that in Each Adherent Normal Tissue
}

\author{
Yoko Matsuzawa, Takumi Kitamoto, Sachiko Suematsu, Jun Saito, Masao Omura and Tetsuo Nishikawa* \\ Endocrinology and Diabetes Center, Yokohama Rosai Hospital, Yokohama, Japan
}

Received: January 13, 2014; Accepted: February 04, 2014; Published: February 06, 2014

“Corresponding author: Tetsuo Nishikawa, Endocrinology and Diabetes Center, Yokohama Rosai Hospital, 3211 Kozukue-cho, Kohoku-ku, Yokohama City, Kanagawa 222-0036, Japan, Tel: 81-454748111; Fax: 81-454748323; E-mail: tetsuon@yokohamah.rofuku.go.jp

\begin{abstract}
Introduction: The steroidogenic activity in a KCNJ5-gene mutated aldosterone-producing adenoma (APA) was reported to be much stronger than that in an APA of the wild type, while it is still controversial.
\end{abstract}

Objectives: We examined whether the normal adrenal tissue attached to APA also possesses KCNJ5 gene mutations, and also investigated steroidogenic activity of mutated and non-mutated APA tissue cells with that of each normal tissue cell attached to APA in order to clarify the nature of steroidogenic activity.

Methods: We selected 12 patients with and without KCNJ5 gene mutation-harboring APA in whom normal adrenal tissue adhered to the APA. We analyzed the presence of KCNJ5 gene mutations by using those tissues.

Results: APA tissues of 6 patients possessed KCNJ5 gene mutations, whereas those mutations were not detected in any of the adjacent normal tissue samples as well as another 6 cases of APA. In two APA tissues with or without KCNJ5 gene mutation, we also compared the steroidogenic activity of isolated cells from each APA tissue with that from the normal adherent tissues. The cortisol production of the mutated APA cells was markedly decreased compared with that of the normal cells, suggesting that the aldosterone production pathway becomes dominant and the cortisol production pathway is downregulated in that APA tissue. Furthermore, the ratio of aldosterone to cortisol in the normal adherent tissue was about $1 / 4^{\text {th }}$ of that of the wild-type APA tissue, while it was about $1 / 10^{\text {th }}$ in the mutated APA.

Conclusion: KCNJ5 gene mutations in the normal tissue adhering to KCNJ5 gene mutation-harboring APA was not observed, suggesting that somatic mutations of the KCNJ5 gene only occur in APA tissue. It is also suggested that KCNJ5 gene mutations dominantly induce the aldosterone production pathway as well as concomitant decline in cortisol production, resulting in highly autonomous aldosterone production.

Keywords: Aldosterone-producing adenoma; KCNJ5 gene; Adherent normal tissue; Aldosterone; Cortisol

\section{Introduction}

Two recurrent somatic mutations in and near the gene for the selectivity filter of the potassium $(\mathrm{K}+)$ channel KCNJ5 were recently detected in aldosterone-producing adenomas (APA). These mutations were reported to produce increased sodium $(\mathrm{Na}+)$ conductance and cell depolarization resulting in autonomous aldosterone production in the APA [1]. In a recent study of Japanese patients with APA, it was reported that 15 $(65.2 \%)$ of the 23 APA had two somatic mutations in their KCNJ5 genes [2], suggesting that KCNJ5 gene mutations are closely related to the pathogenesis of APA in Japanese primary aldosteronism (PA) patients [2]. Thus, we tried to compare clinical characteristics of KCNJ5 gene mutation-harboring APA, with those of APA with no mutations. Furthermore, we examined whether the normal adrenal tissue attached to APA also possesses KCNJ5 gene mutations, and also investigated steroidogenic activity of mutated and non-mutated APA tissue cells with that of each normal tissue cells attached to APA in order to directly clarify the nature of steroidogenic activity for synthesizing aldosterone and cortisol by using isolated cells prepared from each APA and normal tissue.

\section{Subjects and Methods}

Tissue specimens from 8 (Cases 1 8) and 4 patients (Cases 9 12) with APA, who underwent unilateral total and partial resection of the affected adrenal gland respectively, were analyzed (Table 1). We could use the removed adrenal tissues containing adenoma with normal adherent tissue in Cases 1 8 for genetic analysis because of unilateral total adrenalectomy, while could use only APA tissues in Cases 9 12 due to partial resection of the affected adrenal gland. Furthermore, tissue samples were too limited to prepare isolated cells for studying steroidogenesis described below. Thus, we only used tissue samples from Case 2 and 7 since those cases were female, demonstrating almost the same values of PAC and PRA as shown in Table 1. The subjects were definitively diagnosed with APA during the screening of hypertensive patients who visited our general outpatient clinic between January 2013 and June 2013 according to the 
guidelines developed by the Japan Endocrine Society (JES) [3]. The clinical findings of 12 subjects were shown in Table 1 , and we could definitely localize affected adrenals by adrenal vein sampling as depicted in Table 2 . The diagnosis of primary aldoteronism was confirmed by endocrinological examinations such as the furosemide plus upright test, saline-loading test, and captopril-loading test [3]. Computed tomography scans and adrenocorticotropic hormone-loading adrenal vein sampling (ACTH-AVS) were performed to determine the laterality of each patient's hyperaldosteronism, as reported previously [4,5]. All of the patients displayed obvious unilateral hyperaldosteronism and underwent unilateral total resection of the affected adrenal gland. In addition, they all exhibited postoperative improvements in their endocrinological abnormalities, such as hyporeninemic hyperaldosteronemia, and blood pressure (data not shown). The present study was approved by the research ethics committee of Yokohama Rosai Hospital, and all of the subjects understood the objectives of the study and gave their written consent.

\section{RNA extraction and detection of mutations in KCNJ5 cDNA by PCR and direct sequencing}

The specimens of APA tissue and the normal adrenal tissue that had been separated from each APA were separately frozen in liquid nitrogen immediately after their removal during surgery and were stored at $-80{ }^{\circ} \mathrm{C}$. Total RNA was prepared from each tissue using the TriPure Isolation Reagent and High Pure RNA Isolation kit (Roche Diagnostics), as reported previously [6-8]. Then, cDNA was reverse-transcribed from the total RNA using the High Capacity cDNA Reverse Transcription Kit (Applied Biosystems). To sequence the KCNJ5 cDNA, PCR was performed using $1.0 \mu \mathrm{l}$ cDNA together with Tks Gflex $^{\mathrm{TM}}$ DNA Polymerase (Takara-bio) and the following primers: forward primer: 5'-ACCTGGACCATGTTGGCGACC-3', reverse primer: 5'-TCCCGCATGGAGATGACTGCG-3'. The PCR products (286 bp., positions 664 949 NM_000890.3) were purified by electrophoresis (2\% agarose gel) and NucleoSpin Gel and PCR Clean-up (MACHEREY-NAGEL GmbH \& Co.KG), before being directly sequenced by a custom service (Premix sequence, Takara-bio.). All experiments were repeated at least twice.

\section{Preparation of isolated adrenal cells and assessment of steroidogenesis}

Isolated adrenal adenoma cells or adherent normal adrenal cells were soon prepared after their removal during surgery as described previously $[9,10]$ and then resuspended in KrebsRinger bicarbonate buffer ( $\mathrm{pH} 7.4$ ) containing $1.3 \mathrm{mM} \mathrm{CaCl}_{2}, 0.2 \%$ glucose, $0.1 \%$ bovine serum albumin, and 20 mM HEPES (KRBGAHEPES). Cell numbers were determined by the CyQUANT cell proliferation assay kit (Molecular Probes, Inc., Eugene, OR, USA). More than $98 \%$ of the dispersed cells were viable according to the trypan blue dye exclusion test. Next, the adrenal cells were re-suspended at a final density of $10 \times 10^{4}$ cells/ml in KRBGAHEPES containing $1.3 \mathrm{mM} \mathrm{CaCl}_{2}$ and incubated for 120 min under constant agitation at $37^{\circ} \mathrm{C}$ after 15 min pre-incubation with the same buffer. The aldosterone and cortisol concentrations of the medium were measured as reported previously $[6,7,9,10]$.

\section{Statistical analysis}

All results are expressed as the mean \pm SD from at least three different experiments/measurements. We have used three different portions of the APA and its adherent normal adrenal tissues of Cases 2 and 7 for analyzing steroidogenesis. Student's $t$-test was used for comparisons. Differences were considered significant if the $p$-value was less than 0.05 .

\section{Results}

\section{Clinical characteristics}

Age, PAC, PRA, ARR and serum potassium level in APA patients with KCNJ5 gene mutations (Cases 1 6) were $44 \pm 7$ years old, $510 \pm 578 \mathrm{pg} / \mathrm{ml}, 0.17 \pm 0.10 \mathrm{ng} / \mathrm{dl} / \mathrm{hr}, 4756 \pm 6045$ and $2.8 \pm 0.5$

Table 1: Clinical characteristics of subjects with aldosterone-producing adenoma.

\begin{tabular}{|c|c|c|c|c|c|c|c|c|}
\hline Case\# & Age & Gender & PAC & PRA & ARR & $\mathbf{K}$ & $\begin{array}{c}\text { Dexa Suppression test } \\
\text { (cortisol, } \mu \mathrm{g} / \mathrm{dl} \text { ) }\end{array}$ & $\begin{array}{c}\text { Unilateral } \\
\text { adrenalectomy }\end{array}$ \\
\hline 1 & 56 & female & 120 & 0.1 & 1200 & 2.8 & 1.8 & Right adrenal \\
\hline 2 & 41 & female & 738 & $<0.1$ & $>7380$ & 2.7 & 1.3 & Left adrenal \\
\hline 3 & 43 & male & 192 & 0.3 & 640 & 3.5 & 2.1 & Left adrenal \\
\hline 4 & 36 & female & 1590 & $<0.1$ & $>15900$ & 2.1 & 0.9 & Right adrenal \\
\hline 5 & 46 & female & 303 & 0.1 & 3030 & 2.6 & 1.0 & Right adrenal \\
\hline 6 & 41 & female & 116 & 0.3 & 386.6 & 3.2 & 0.8 & Right adrenal \\
\hline 7 & 61 & female & 953 & 0.2 & 47650 & 3.5 & 1.7 & Right adrenal \\
\hline 8 & 61 & female & 127 & 0.6 & 212 & 3.8 & 0.9 & Right adrenal \\
\hline 9 & 62 & female & 235 & 0.1 & 2350 & 3.3 & 1.7 & Left adrenal \\
\hline 10 & 62 & female & 172 & $<0.1$ & $>1720$ & 3.4 & 2.5 & Right adrenal \\
\hline 11 & 49 & female & 209 & 0.2 & 1045 & 2.8 & 1.4 & Left adrenal \\
\hline 12 & 29 & female & 213 & 0.2 & 1065 & 2.9 & 1.9 & Right adrenal \\
\hline
\end{tabular}

Cases 1 to 6 showed KCNJ5 gene mutations in their aldosterone-producing adenomas, while cases 7 to 12 did not show any mutations.

PAC- Plasma Aldosterone Concentration; PRA- Plasma Renin Activity; ARR- Aldosterone-Renin Ratio; K- serum potassium level; Dexa suppressionovernight $1 \mathrm{mg}$ dexamethasone suppression test; AVS- Adrenal Vein Sampling; n.p.- not performed due to allergy against a contrast medium. 
mEq/L, respectively (Table 1). Age, PAC, PRA, ARR and serum potassium level in APA patients without KCNJ5 gene mutations (Cases 7 12) were $54 \pm 13$ years old, $318 \pm 313 \mathrm{pg} / \mathrm{ml}, 0.23 \pm 0.19$ $\mathrm{ng} / \mathrm{dl} / \mathrm{hr}, 9007 \pm 18945$ and $3.3 \pm 0.4 \mathrm{mEq} / \mathrm{L}$, respectively. There was a significant difference in serum potassium level between two groups $(\mathrm{p}<0.05)$. PAC and PRA were higher and lower in the mutated group than the wild group, respectively, while there was statistically no significant difference between two groups. Highest value of PAC in right or left adrenal vein sampling in the mutated group and the wild type group was $67038 \pm 81269 \mathrm{pg} / \mathrm{ml}$ and $74383 \pm 45736 \mathrm{pg} / \mathrm{ml}$, respectively without any statistically significant difference. Moreover, the ratio of PAC to cortisol in the affected adrenal vein in the mutated group and the wild type group was $117.2 \pm 170$ and $96.1 \pm 43.0$, respectively without any statistically significant difference (Table 2).

\section{KCNJ5 gene mutations}

No KCNJ5 gene mutations were noted in the adjacent normal tissues $(\mathrm{N})$ in any of the 8 APA patients with or without KCNJ5 gene mutations, although the APA tissues in cases 1 to 6 demonstrated KCNJ5 gene mutations and those in cases 7 to 12 did not (Table 3 ). We could not analyze KCNJ5 gene mutations in $\mathrm{N}$ of cases 9 to 12, because we did not remove the adherent normal portion of adrenals by partial adrenalectomy.

\section{Steroidogenesis}

APA and adherent normal adrenal cells were isolated from two of the above patients (Cases $2 \& 7$ ) and cultured. Case 2 demonstrated that the aldosterone level of the APA culture supernatant was about 1.4 times higher than that of the normal adrenal cell culture supernatant (Figure 1A). Furthermore, the APA cells secreted 1/10th of the amount of cortisol secreted by the normal cells (Figure 1B). Thus, in case 2 the aldosterone to cortisol ratio of the normal tissue was almost $1 / 10$ th of that of the APA tissue (Figure 1C). On the other hand, in Case 7 which do not harbor KCNJ5 gene mutation, the amount of aldosterone was almost twice higher in the APA tissue cells than that of the normal adherent tissue cells (Figure 2A). The cortisol production by the APA tissue cells of Case 7 was $6 / 10^{\text {th }}$ of that by the normal adherent tissue cells (Figure 2B). Then, the ratio of aldosterone to cortisol in the normal adherent tissue of Case 7 was almost $1 / 4^{\text {th }}$ of that of the APA tissue (Figure 2C). Aldosterone production by isolated cells of the adenoma tissue in Case 2 was significantly lower than that in Case 7 ( $\mathrm{p}<0.02)$, while cortisol production by isolated cells of the adenoma tissue in Case 2 was not significantly differed from that in Case 7. Aldosterone production by isolated cells of the adherent normal tissue in Case 2 was significantly higher than that in Case 7 ( $\mathrm{p}<0.05)$, while cortisol production by isolated cells of the adherent normal tissue in Case 2 was significantly higher than that in Case $7(p<0.05)$. The ratio of aldosterone to cortisol production in the APA tissue of Case 2 and 7 was $0.05 \pm 0.01$ and $0.08 \pm 0.01$, respectively without significant difference between two cases. The ratio of aldosterone to cortisol production in the adherent normal tissue of Case 2 was significantly lower than that in Case $7(\mathrm{p}<0.01)$.

\section{Discussion}

In the present study, whilst KCNJ5 gene mutations were detected in the APA tissues of cases 1 to 6 they were not detected in any of the associated normal adherent adrenal tissue samples. These findings were consistent with previous studies in which KCNJ5 gene mutations were not found in the control tissue samples, which were obtained from the normal adrenal gland tissue adjacent to the APA [2]. Therefore, it seems that KCNJ5 gene mutations only occur in APA tissue, and it is unlikely that APA is caused by gene aberrations being present in all of the cells of the adrenal gland. It was also reported that significantly lower levels of the potassium channel GIRK4 were detected in KCNJ5

Table 2: Adrenal vein sampling.

\begin{tabular}{|c|c|c|c|c|c|c|}
\hline \multirow[b]{2}{*}{ Case\# } & \multicolumn{6}{|c|}{ ACTH-stimulated AVS } \\
\hline & $\begin{array}{c}\text { Laterality of } \\
\text { adrenal adenoma }\end{array}$ & Right & Left & Right & Left & LR \\
\hline 1 & Right & 1580 & 637 & 51400 & 6160 & 3.35 \\
\hline 2 & Left & n.p. & n.p. & n.p. & n.p. & n.p. \\
\hline 3 & Left & $371^{*}$ & $497^{*}$ & $1560 \%$ & $6790 \%$ & 3.26 \\
\hline 4 & Right & 500 & 491 & 209000 & 3410 & 60.1 \\
\hline 5 & Right & 535 & 728 & 44200 & 2880 & 20.9 \\
\hline 6 & Right & 607 & 626 & 23800 & 10000 & 2.45 \\
\hline 7 & Right & 1000 & 683 & 117000 & 2690 & 2.97 \\
\hline 8 & Right & 1070 & 613 & 26100 & 10900 & 1.37 \\
\hline 9 & Left & 864 & 902 & 11500 & 127000 & 10.58 \\
\hline 10 & Right & 388 & 881 & 36900 & 3040 & 27.56 \\
\hline 11 & Left & 776 & 526 & 2090 & 37300 & 26.33 \\
\hline 12 & Right & 796 & 540 & 102000 & 2030 & 34.09 \\
\hline
\end{tabular}

Each value in adrenal vein sampling (AVS) was described as the value after ACTH-stimulation, while the values of PAC $\%$ in case 3 was expressed as the basal value without ACTH-stimulation. Case 2 clearly demonstrated the left adrenal tumor by CT images and left adrenalectomy was done after talking informed consent. A unilateral lesion can be detected if the lateralized ratio (L.R.) (the adrenal vein aldosterone/cortiso; (A/C) ratio of the high-value side)/(that of the low-value side) is 2.6 after ACTH stimulation or if aldosterone is more than $14000 \mathrm{pg} / \mathrm{ml}$ in the affected adrenal side after ACTH stimulation, according to the guideline [3]. 
Table 3: KCNJ5 gene mutations in each subject.

\begin{tabular}{|c|c|c|c|}
\hline \multirow[b]{2}{*}{ Case\# } & \multicolumn{3}{|c|}{ KCNJ5 Gene analysis } \\
\hline & & Codon 151 (p.G151R) & Codon 168 (p.L168R) \\
\hline \multirow[b]{2}{*}{1} & A & G G G & $\mathrm{C} \mathrm{T} / \mathrm{G} \mathrm{C}$ \\
\hline & $\mathrm{N}$ & G G G & C T C \\
\hline \multirow{2}{*}{2} & A & G G G & $\mathrm{C} \mathrm{T} / \mathrm{G} \mathrm{C}$ \\
\hline & $\mathrm{N}$ & G G G & C T C \\
\hline \multirow{2}{*}{3} & A & G G G & $\mathrm{C} \mathrm{T} / \mathrm{G} \mathrm{C}$ \\
\hline & $\mathrm{N}$ & G G G & C T C \\
\hline \multirow{2}{*}{4} & A & G / A G G & C T C \\
\hline & $\mathrm{N}$ & G G G & $\mathrm{C} \mathrm{T} \mathrm{C}$ \\
\hline \multirow{2}{*}{5} & A & G G G & $\mathrm{C} \mathrm{T} / \mathrm{G} \mathrm{C}$ \\
\hline & $\mathrm{N}$ & G G G & C T C \\
\hline \multirow{2}{*}{6} & A & $G /(A) G G$ & C T C \\
\hline & $\mathrm{N}$ & G G G & $\mathrm{C} \mathrm{T} \mathrm{C}$ \\
\hline \multirow{2}{*}{7} & A & G G G & $\mathrm{C}$ T C \\
\hline & $\mathrm{N}$ & G G G & C T C \\
\hline \multirow{2}{*}{8} & A & G G G & C T C \\
\hline & $\mathrm{N}$ & G G G & $\mathrm{C}$ T C \\
\hline \multirow{2}{*}{9} & $\mathrm{~A}$ & G G G & C T C \\
\hline & $\mathrm{N}$ & No data & No data \\
\hline \multirow{2}{*}{10} & A & G G G & C T C \\
\hline & $\mathrm{N}$ & No data & No data \\
\hline \multirow{2}{*}{11} & $\mathrm{~A}$ & G G G & C T C \\
\hline & $\mathrm{N}$ & No data & No data \\
\hline \multirow{2}{*}{12} & A & G G G & $\mathrm{C} \mathrm{T} \mathrm{C}$ \\
\hline & $\mathrm{N}$ & No data & No data \\
\hline
\end{tabular}

Mutations of KCNJS gene in adrenal tissues were analyzed as described in detail in the text.

A- adenoma tissue, $\mathrm{N}$ - normal adrenal tissue adhered to the adenoma
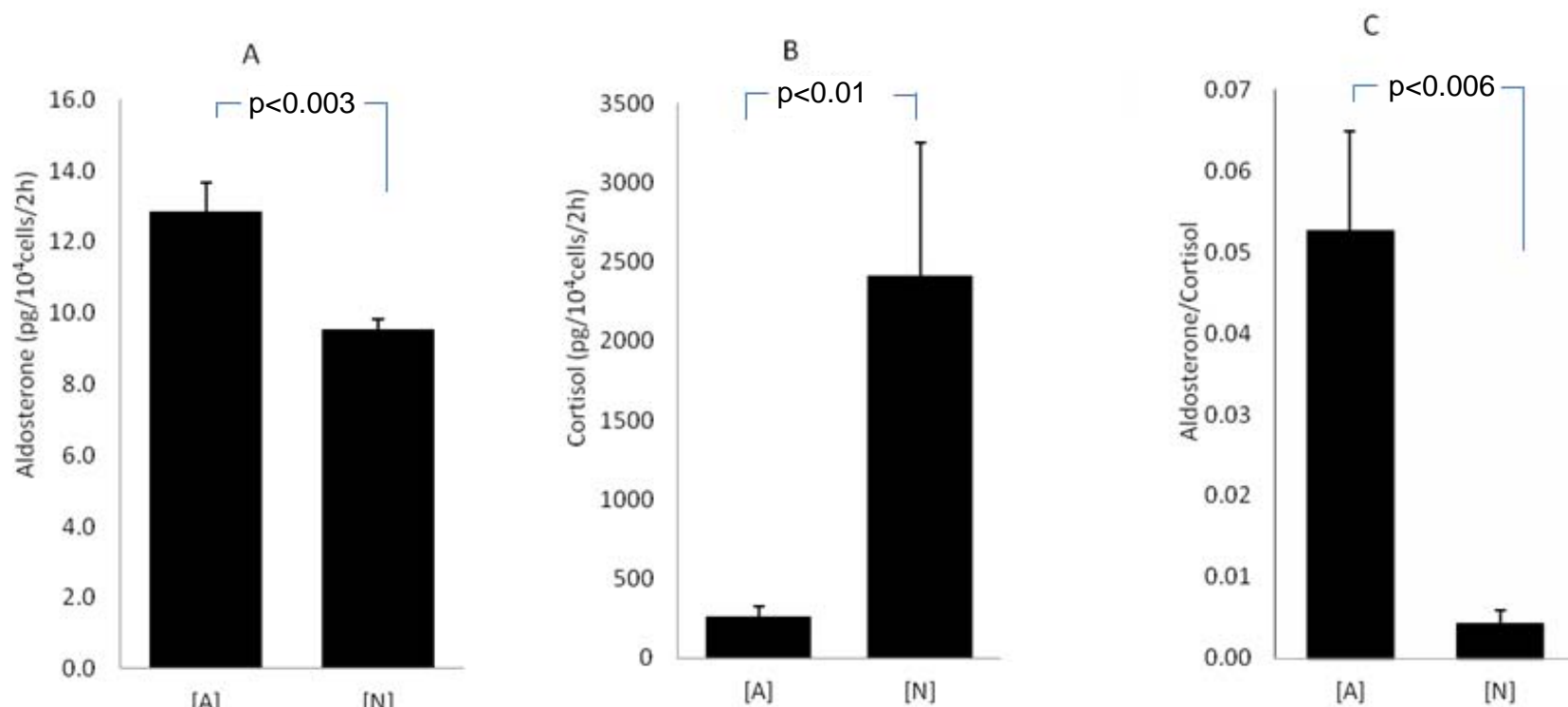

Figure 1: Steroidogenic activity in the APA cells and normal adherent adrenal cells in case 2

A) Aldosterone production by APA cells and normal adherent adrenal cells that had been cultured for 2 hours as described in the text. Results were expressed as mean \pm SD. $p<0.003$; adenoma vs. normal

B) Cortisol production by APA cells and normal adherent adrenal cells that had been cultured for 2 hours as described in the text. Results were expressed as mean \pm SD. $p<0.01$; adenoma vs. normal

C) The ratio of aldosterone to cortisol in the APA cells and normal adherent adrenal cells after 2 hours' incubation.

Results were expressed as mean \pm SD. $p<0.006$; adenoma vs. normal

A- the APA tissue of case 2; $\mathrm{N}$ - the normal adherent adrenal tissue 

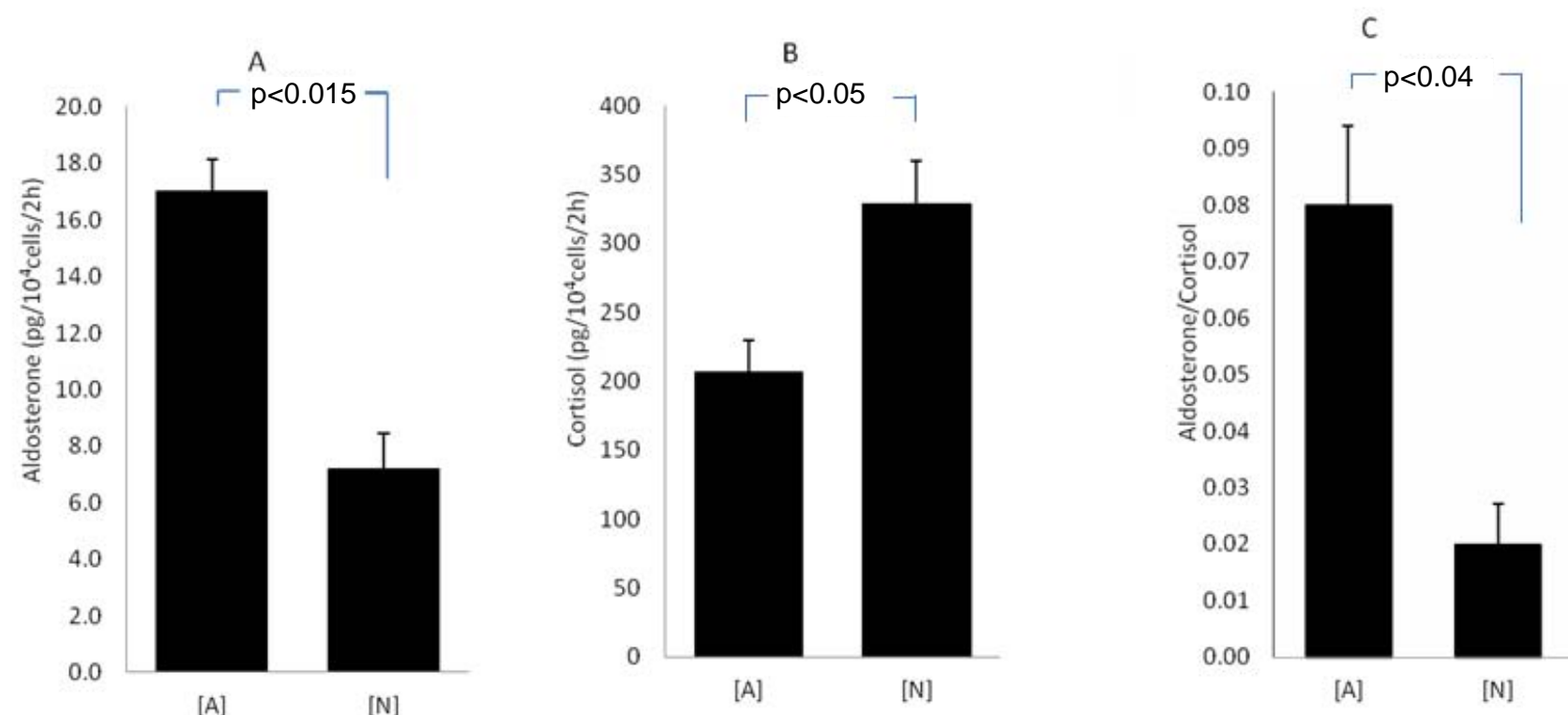

Figure 2: Steroidogenic activity in the APA cells and normal adherent adrenal cells in case 7

A) Aldosterone production by APA cells and normal adherent adrenal cells that had been cultured for 2 hours as described in the text.

Results were expressed as mean \pm SD. $p<0.015$; adenoma vs. normal

B) Cortisol production by APA cells and normal adherent adrenal cells that had been cultured for 2 hours as described in the text.

Results were expressed as mean \pm SD. $p<0.05$; adenoma vs. normal

C) The ratio of aldosterone to cortisol in the APA cells and normal adherent adrenal cells after 2 hours' incubation.

Results were expressed as mean \pm SD. $p<0.04$; adenoma vs. normal

A- the APA tissue of case $7 ; \mathrm{N}$ - the normal adherent adrenal tissue

mutation-harboring APA, although the channel was expressed in APA tissue and the zona glomerulosa of the adjacent cortex [11]. On the other hand, no previous studies have compared the steroidogenic activity of APA and normal tissue by using isolated cells prepared from the removed adrenal tissues. Thus, we compared the amounts of aldosterone and cortisol secreted from APA cells with and without harboring KCNJ5 gene mutations with those secreted by normal adrenal cells from the region adjacent to each APA tissue (Cases 2 \& 7). Aldosterone production when incubated isolated cells of the adenoma tissue of Case 2 with KCNJ 5 gene mutation was not higher than that when incubated isolated cells of the adenoma tissue of Case 7 with no mutation. Thus, it is suggested that the activity of basal aldosterone synthesis by the cells of the KCNJ5 gene mutatied APA tissue seems to be not so strong, comparing with that in the non-mutated APA tissue. On the contrary, the present experiments clearly demonstrated that the cortisol production of the APA cells was markedly decreased compared with that of the adjacent normal cells in Case 2, suggesting that the aldosterone production pathway becomes dominant and the cortisol production pathway is downregulated in the APA tissue, and resulting in autonomous aldosterone production by the cells of the KCNJ5 gene mutated APA tissue. Case 7 was still possessing autonomous ability of aldosterone prodution, while the ratio of aldosterone to cortisol in the normal adherent tissue was almost $1 / 4^{\text {th }}$ of that of the APA tissue. Thus, it is suggested that isolated cells prepared from the APA tissue harboring KCNJ5 gene mutation can synthesize more dominantly aldosterone than cortisol compared with the cells prepared from the APA tissue without harboring KCNJ5 gene mutation. On the other hand, it was reported that the non-tumor portions of the microadenomas showed marked and sustained CYP11B2 mRNA expression under the suppressed renin-angiotensin system [12]. Thus it is suggested that the normal adrenal portion adhered to APA possesses the ability of aldosterone synthesis, and also that steroidogenic activity of cortisol and aldosterone production in the normal adherent tissue may be differently influenced by functional characteristics of steroidogenesis of each APA tissue.

This observation is consistent with our previously reported findings regarding APA tissue [8]. CYP11B2 and CYP17 are key enzymes of the aldosterone and cortisol pathways, respectively. In the abovementioned study, we detected a low CYP17/HSD3B2 ratio in APA tissue, indicating that the cortisol pathway is suppressed in APA. In addition, we also observed a high CYP11B2/ CYP11B1 ratio in APA tissue, suggesting that the aldosterone pathway is accelerated in APA; however, it was unclear whether the examined APA tissues possessed KCNJ5 gene mutations. The results of the present study also agree with those reported by Stowasser M [13], who found that KCNJ5 mutations caused reduced $\mathrm{K} / \mathrm{Na}$ channel selectivity and $\mathrm{Na}$ influx, and hence, induced increases in the cell membrane depolarization, calcium influx, aldosterone synthesis-promoting gene expression, and aldosterone production of adrenocortical cells. Moreover, it was reported that KCNJ5 mutations are associated with young age, female gender and a more florid hyperaldosteronism with higher preoperative aldosterone and/or lower plasma K levels [2,14- 
17]. From the present data of clinical findings, the mutated group demonstrated significantly lower plasma $\mathrm{K}$ levels than the wild type group, and also the mutated group showed not significant but higher tendency of PAC than the wild type group. Thus, our clinical data are consistent with the previous findings [2,1417]. Moreover, the present results by using isolated cells from the APA tissues with and without KCNJ5 gene mutation clearly demonstrated the higher ability of synthesizing aldosterone rather than cortisol in KCNJ5 mutation-harboring APA than that in the APA tissue of the wild type, even though the amount of basal aldosterone production was slightly lower in the mutated group than in the wild type group. Those data on in vitro study also are consistent with clinical findings as reported previously [2,14-17].

\section{Conclusion}

We did not detect KCNJ5 gene mutations in the normal tissue adhering to KCNJ5 gene mutation-harboring APA, suggesting that somatic mutations of the KCNJ5 gene only occur in APA tissue. We need to conduct further studies involving larger numbers of adrenal tissue samples obtained from patients with PA, although it is quite difficult to obtain sufficient amounts of APA and adherent normal tissue. On the other hand, the present results clearly demonstrate that APA cells produce markedly less cortisol than normal adrenal cells, and it is suggested that KCNJ5 gene mutations dominantly induce the aldosterone production pathway as well as a concomitant decline in cortisol production, resulting in highly autonomous aldosterone production.

\section{Acknowledgement}

This study was partly supported by a Grant for Research on Intractable Diseases from the Japanese Ministry of Health, Labour and Welfare.

\section{References}

1. Choi M, Scholl UI, Yue P, Bjorklund P, Zhao B, et al. (2011) K+ channel mutations in adrenal aldosterone-producing adenomas and hereditary hypertension. Science 331: 768-772.

2. Taguchi R, Yamada M, Nakajima Y, Satoh T, Hashimoto K, et al. (2012) Expression and mutations of KCNJ5 mRNA in Japanese patients with aldosterone-producing adenomas. J Clin Endocrinol Metab 97: 13111319.

3. Nishikawa T, Omura M, Satoh F, Shibata H, Takahashi K, et al. (2011) Guidelines for the diagnosis and treatment of primary aldosteronism-the Japan Endocrine Society 2009. Endocr J 58: 711-721.

4. Omura M, Sasano H, Fujiwara T, Yamaguchi K, Nishikawa T (2002) Unique cases of unilateral hyperaldosteronemia due to multiple adrenocortical micronodules, which can only be detected by selective adrenal venous sampling. Metabolism 51: 350-355.
5. Omura M, Sasano H, Saito J, Yamaguchi K, Kakuta Y, et al. (2006) Clinical characteristics of aldosterone-producing microadenoma, macroadenoma, and idiopathic hyperaldosteronism in 93 patients with primary aldosteronism. Hypertens Res 29: 883-889.

6. Nishikawa T, Suematsu S, Saito J, Soyama A, Ito H, (2005) Human renal mesangial cells produce aldosterone in response to low-density lipoprotein (LDL). J Steroid Biochem Mol Biol 96: 309-316.

7. Nishikawa T, Matsuzawa Y, Suematsu S, Saito J, Omura M, et al (2010) Effect of atorvastatin on aldosterone production induced by glucose, LDL or angiotensin II in human renal mesangial cells. Arzneimittelforschung 60: 445-451.

8. Sakuma I, Suematsu S, Matsuzawa Y, Saito J, Omura M, et al. (2013) Characterization of steroidogenic enzyme expression in aldosteroneproducing adenoma: a comparison with various human adrenal tumors. Endocr J 60: 329-336.

9. Yaguchi H, Tsutsumi K, Shimono K, Omura M, Sasano H, et al. (1998) Involvement of high density lipoprotein as substrate cholestero1 for steroidogenesis by bovine adrenal fasciculo-reticularis cells. Life Sciences 62: 1387-1395.

10. Shimono K, Tsutsumi K, Yamaguchi H, Omura M, Sasano H, et al. (1999) Lipoprotein lipase promoting agent,NO-1886,modu1ates adrenal functions-species difference in effects of NO-1886 on steroidogenesis. Steroids 64: 453-459.

11. Boulkroun S, Golib Dzib JF, Samson-Couterie B, Rosa FL, Rickard AJ, et al. (2013) KCNJ5 mutations in aldosterone producing adenoma and relationship with adrenal cortex remodeling. Mol Cell Endocrinol 371: 221-227.

12. Karashima S, Takeda Y, Cheng Y, Yoneda T, Demura M, et al. (2011) Clinical characteristics of primary hyperaldosteronism due to adrenal microadenoma. Steroids 76: 1363-1366.

13. Stowasser M (2013) Primary aldosteronism and potassium channel mutations. Curr Opin Endocrinol Diabetes Obes 20: 170-179.

14. Akerstrom T, Crona J, Delgado Verdugo A, Starker LF, Cupisti K, et al. (2012) Comprehensive re-sequencing of adrenal aldosterone producing lesions reveal three somatic mutations near the KCNJ5 potassium channel selectivity filter. PLoS ONE 7: e41926.

15.Azizan EA, Murthy M, Stowasser M, Gordon R, Kowalski B, et al. (2012) Somatic mutations affecting the selectivity filter of KCNJ5 are frequent in 2 large unselected collections of adrenal aldosteronomas. Hypertension 59: 587-591.

16. Boulkroun S, Beuschlein F, Rossi GP, Golib-Dzib JF, Fischer E, et al. (2012) Prevalence, clinical, and molecular correlates of KCNJ5 mutations in primary aldosteronism. Hypertension 59: 592-598.

17. Monticone S, Hattangady HG, Nishimoto K, Mantero F, Rubin B, et al. (2012) Effect of KCNJ5 mutations on gene expression in aldosteroneproducing adenomas and adrenocortical cells. J Clin Endocrinol Metab 97: E1567-1572. 